Research Article

\title{
Valuing Multirisk Catastrophe Reinsurance Based on the Cox-Ingersoll-Ross (CIR) Model
}

\author{
Wen Chao (iD) \\ School of Management, Fujian University of Technology, Fuzhou 350108, China \\ Correspondence should be addressed to Wen Chao; chaowen2014@163.com
}

Received 15 September 2020; Revised 27 January 2021; Accepted 30 January 2021; Published 11 February 2021

Academic Editor: Polinpapilinho Katina

Copyright (c) 2021 Wen Chao. This is an open access article distributed under the Creative Commons Attribution License, which permits unrestricted use, distribution, and reproduction in any medium, provided the original work is properly cited.

\begin{abstract}
Catastrophe risks lead to severe problems of insurance and reinsurance industry. In order to reduce the underwriting risk, the insurer would seek protection by transferring part of its risk exposure to the reinsurer. A framework for valuing multirisk catastrophe reinsurance under stochastic interest rates driven by the CIR model shall be discussed. To evaluate the distribution and the dependence of catastrophe variables, the Peaks over Threshold model and Copula function are used to measure them, respectively. Furthermore, the parameters of the valuing model are estimated and calibrated by using the Global Flood Date provided by Dartmouth College from 2000 to 2016. Finally, the value of catastrophe reinsurance is derived and a sensitivity analysis of how stochastic interest rates and catastrophe dependence affect the values is performed via Monte Carlo simulations. The results obtained show that the catastrophe reinsurance value is the inverse relation between initial value of interest rate and average interest rate in the long run. Additionally, a high level of dependence between catastrophe variables increases the catastrophe reinsurance value. The findings of this paper may be interesting to (re)insurance companies and other financial institutions that want to transfer catastrophic risks.
\end{abstract}

\section{Introduction}

Nowadays, catastrophe events, such as floods, earthquakes, hurricanes, storms, and man-made disasters claiming many lives and causing great property loss, are of low-probability but relatively great destructiveness. For example, Hurricane Andrew in the US caused economic losses of 30 billion in 1992 and at least 69,000 people were killed in Wenchuan earthquake that occurred in Sichuan Province of China. What is worse is that, in recent years, the catastrophe events in terms of both frequency and magnitude reveal a rising trend. These severe and unpredictable catastrophic losses place enormous financial stress on insurance businesses and even might lead them to bankruptcy. In order to hedge and transfer the catastrophe risks, insurance companies need to purchase catastrophe exceeding loss cover from reinsurers.

Reinsurers can help the insurers reduce the underwriting risks. A key question is how one should value the reinsurance contracts. Many papers have been conducted for the valuing of catastrophe reinsurance. The early valuing model is due to Strickler [1] (see also [2]), in which a constant deterministic rate of catastrophes is assumed, and it is limited to catastrophes claiming at most 1500 lives. To overcome these shortcomings of the valuing model used in [1], Ekheden and Hössjer [3] established a new model based on compound Poisson process to value the catastrophe excess of loss cover. Another common technique applied to value reinsurance contracts is based on simulation approach; for example, see [4-7] and the references therein. Particularly, Leppisaari [4] improved the method applied in [3] and suggested a microsimulation to value life catastrophes. In [7], Daykin et al. proposed a so-called frequency severity method that uses the technology of simulating for both the number and size of individual claims.

We also point out that catastrophe bond is an innovative financial instrument, by which the insurers transfer the risk of a possible large payment created by catastrophic events to the capital market. Applying stochastic control theory and Hamilton-Jacobi-Bellman equations, Egami and Young [8] studied the optimal proportional reinsurance for reinsurer. 
Based on this result and the principle of equivalent utility, the authors further obtained the indifference price of catastrophe bond. Giuricich and Burnecki [9] calculated the value of catastrophe bond under a left-truncated heavytailed model. In [10], Romaniuk analysed the behavior of insurance portfolio containing catastrophe bond by using a fuzzy number to model its value. For more information on this direction, we refer the interested readers to [11-14] and the references therein.

As we can see from the works described above, the existing literatures mainly focused on the univariate catastrophe reinsurance valuing. However, one catastrophe accident frequently causes not just one kind of claim in our life. In [15], Chan et al. described situations where an unexpected claim event might induce more than one type of claim. In that article, a typical example of motor insurance was shown, in which an accident could cause claims for vehicle damage and bodily injuries. Clearly, a similar phenomenon also exists in natural catastrophe insurance. Up to now, there have been a number of ruin theory actuaries studying multirisk claims [16-20]. As far as we know, there has not been any research on valuing of catastrophe reinsurance of multirisk claims, which is just our purpose here. In addition, most prior studies for catastrophe reinsurance valuing did not take into account the factor of the interest rate.

Compared with previous works, in the present paper, our main contributions to the field of catastrophe reinsurance valuing are twofold. First, in order to develop a framework for valuing of multirisk catastrophe reinsurance contract, we use Copula function to describe the dependence of catastrophe variables. This method is utilized for the first time in the catastrophe reinsurance contract area. Second, we apply the Cox-Ingersoll-Ross (CIR) model to depict the characteristics of interest rates market, thereby providing more accurate value of catastrophe reinsurance contract.

The remainder of the paper is organized as follows: Section 2 briefly describes the framework of CAT claim model and stochastic interest model. Section 3 presents an empirical analysis. Section 4 is devoted to Monte Carlo simulation and sensitivity analysis. Finally, Section 5 offers conclusions for this research.

\section{Valuation Framework}

2.1. Modeling Assumptions. We consider a catastrophe reinsurance contract with maturity $T$ years. Let $\left\{X_{i}, i=\right.$ $1,2, \ldots\}$ and $\left\{Y_{i}, i=1,2, \ldots\right\}$ be two sequences of independent and identical distribution random variables with cumulative distribution functions $F_{X}$ and $F_{Y}$, respectively. We treat $X_{i}$ and $Y_{i}$ as the values of two kinds of catastrophic losses during the $i$-th catastrophic event.Let $\{N(t), t \geq 0\}$ be a Poisson process with intensity $\lambda>0$. For each $t \in[0, T]$, the value of the process $N(t)$ is equal to the number of catastrophic events until the moment $t$. Assume that $\{N(t)\}$, $\left\{X_{i}\right\}$, and $\left\{Y_{i}\right\}$ are mutually independent.
2.2. Valuation Theory. By the fundamental theorem of asset pricing, the expected catastrophe reinsurance value $P$ can be given as

$$
P=E\left[\sum_{i=1}^{N(T)} f\left(X_{i}, Y_{i}\right) \exp \left(-\int_{0}^{t_{i}} r(s) \mathrm{d} s\right)\right] .
$$

Here, $r(s)$ denotes a single-factor spot interest rate, $f\left(X_{i}, Y_{i}\right)=\max \left\{c_{1} X_{i}+c_{2} Y_{i}-D, 0\right\}$ is generally called the ceded loss function (coverage function) in the literature, while $D$ denotes the catastrophe reinsurance attachment point, and $c_{1}$ and $c_{2}$ are the claim coefficients. It then follows that $\sum_{i=1}^{N(T)} f\left(X_{i}, Y_{i}\right) \exp \left(-\int_{0}^{t_{i}} r(s) \mathrm{d} s\right)$ is exactly the present value of total claims within the period $[0, T]$. However, we have to use the Monte Carlo simulation technique to seek the numerical solution for $P$ due to the fact that the explicit solution is difficult to calculate.

2.3. Interest Rate Model. In the past two decades, economists have put forward a large number of interest rate models, which can be roughly divided into single-factor interest rates and multifactor interest rates. In the study of catastrophe reinsurance, we focus our attention on the effect of interest rate on the reinsurance values. As a consequence, singlefactor interest rate models are selected. In particularly, the basic single-factor interest rate models are Merton model, Vasicek model, and CIR model. The CIR model can not only describe the mean-reverting characteristic of interest rate but also guarantee nonnegative interest rate; see [21]. Meanwhile the Merton model cannot describe the interest rate mean-reverting process, and the Vasicek model cannot ensure that the interest rate is constantly a positive number. Therefore, this paper assumes that the spot interest rate follows the CIR model. This interest rate model can be denoted by the following equation:

$$
\mathrm{d} r(t)=\alpha(\mu-r(t)) \mathrm{d} t+\beta \sqrt{r(t)} \mathrm{d} W(t),
$$

where $\alpha>0$ is the speed of mean-reverting, $\mu>0$ is a mean of interest rate in the long run, $\beta>0$ is the volatility of the interest rate, and $W(t)$ is a standard Brownian process.

2.4. Extreme Value Theory and Modeling. In this subsection, we will apply extreme value theory to depict the heavy tail property of extreme events related to historical observations. Specifically, extreme value theory is mainly used to study the extreme value distribution and its characteristics of random variables. It has outstanding pertinence to the heavy-tailed distribution. In particular, one of its advantages is that the extreme theory does not require any assumption on the overall distribution, and it directly uses sample data to deduce the tail characteristics of distribution. There are two main extreme models in the practical application of finance and insurance claims. One is called the Generalized Extreme Value (GEV) distribution, which focuses on the statistical 
behaviour of maxima within one group. However, the greatest drawback of GEV approach is that it is terribly wasteful with observations, especially considering the fact that the extreme events are rare by definition. Some data that are possibly informative about the extreme behaviour of the process are discarded completely in the GEV model. The other model of extreme theory is called Generalized Pareto Distribution (GPD) (see [22]). A great improvement of this model is to take into consideration not only the maxima but also more other extreme observations. Hence, the GPD model incorporates more data into the inference and less observations are omitted. For the sake of this, we choose the GPD model to analyse the catastrophe events in this paper.

Let $x^{*}$ denote the upper end-point of $F$. In the case of GPD model, for given large $u<x^{*}$, we call $u$ a threshold and all the observations above the threshold are used in the analysis. Additionally, $X_{i}-u$ is called excess. It is easy to see that

$$
F_{u}(y)=P(X-u \leq y \mid X>u)=\frac{F(u+y)-F(u)}{1-F(u)}, \quad y \geq 0,
$$

which implies that

$$
F(x)=(1-F(u)) F_{u}(x-u)+F(u), \quad x \geq u .
$$

According to Balkema and Pickands [23, 24], for large $u$, the excess distribution $F_{u}(y)$ in equation (4) can be approximated by GPD; that is,

$$
F_{u}(y) \approx G_{\xi, \sigma}(y)= \begin{cases}1-\left(1+\xi \frac{y}{\sigma}\right)^{-1 / \xi}, & \text { if } \xi \neq 0 ; \\ 1-\exp \left(-\frac{y}{\sigma}\right), & \text { if } \xi=0,\end{cases}
$$

where $\xi$ and $\sigma$ are the shape parameter and the scale parameter, respectively.

Then, substituting (5) into (4), we may obtain the following for $x>u$ :

$$
F(x)= \begin{cases}(1-F(u))\left(1-\left(1+\xi \frac{x-u}{\sigma}\right)\right)+F(u), & \text { if } \xi \neq 0 ; \\ (1-F(u))\left(1-\exp \left(-\frac{x-u}{\sigma}\right)\right)+F(u), & \text { if } \xi=0 .\end{cases}
$$

\section{Parameter Estimation of the Valuing Model}

3.1. Data Analysis. We take for our study the Global Archive of Large Flood Events, provided by Dartmouth College. Our focus is the observations of damage areas and deaths in China between 2000 and 2016. Typically, the cases where at least three lives are lost in a single event are often considered to be catastrophe (see [3]). By this way, a total of 126 pairs of observations for damage areas and deaths are picked out.

We need first to detect the tail behaviour of catastrophe loss data before applying extreme value theory. In general, we may use the kurtosis or exponential Q-Q plot of data to judge the heavy-tailed characteristic. Firstly, we find that the kurtoses of damage areas and deaths are 32.088 and 25.478, respectively, which are both larger than three. Secondly, Figure 1 obviously shows the tail of exponential Q-Q plots appearing in a convex shape. Thus, it is reasonable to assume that the sample data are of heavy-tailed character.

3.2. Parameter Estimation of the POT Model. In this subsection, we construct the marginal distributions of the two loss variables and take the case of damage areas as an example for our analysis. The other case of deaths can be discussed in the same way. Recall that the damage areas are denoted by random variable $X$. In the POT model, if the observation values excessed the threshold $u_{X}$, the exceeding part could be described by GPD. For the other observation values, the empirical distribution function is suitable. Thus, we can write the distribution of damage areas as

$$
F_{X}(x)= \begin{cases}\widehat{F}_{X}\left(u_{X}\right)+\left(1-\widehat{F}_{X}\left(u_{X}\right)\right) G_{\xi_{X}, \sigma_{X}}\left(x-u_{X}\right), & \text { if } u_{X} \leq x, \\ \widehat{F}_{X}(x), & \text { if } u_{X}>x\end{cases}
$$

where $\widehat{F}_{X}(\cdot)$ is the empirical distribution function of $X$.

As is known, the threshold selection obviously plays a paramount role in the GPD model. Choosing an appropriate threshold for the threshold model involves a trade between the variance of model and the systematic bias. Taking a larger threshold means fewer data can be adapted in the inference, which leads to larger variance in the estimation. On the other hand, choosing a lower threshold implies that more data are available to estimate in the analysis, consequently decreasing the variance of the results. However, choosing the threshold too low will lead to invalidity of the GPD approximation of the excess distribution. Therefore, how to choose an appropriate threshold for the model is a main challenge. In practical applications, there are two ways to choose threshold. The first way is based on the mean residual plot. Generally, the threshold is justified if the mean excess plot becomes roughly linear, which starts from certain threshold level. For more details, see Embrechts et al.'s work [25]. Figures 2 and 3 display the mean excess plots of catastrophe damage areas and deaths, respectively. From Figure 2, we see 


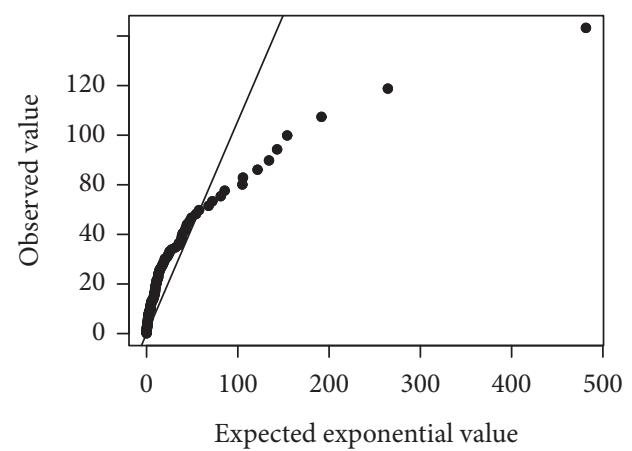

(a)

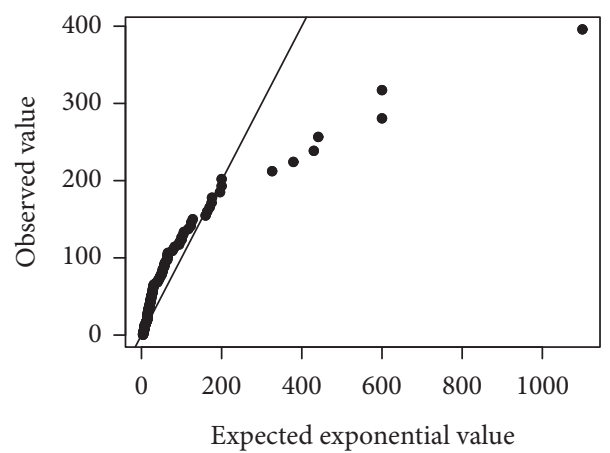

(b)

Figure 1: Exponential Q-Q plot of damage areas (a) and exponential Q-Q plot of deaths (b).

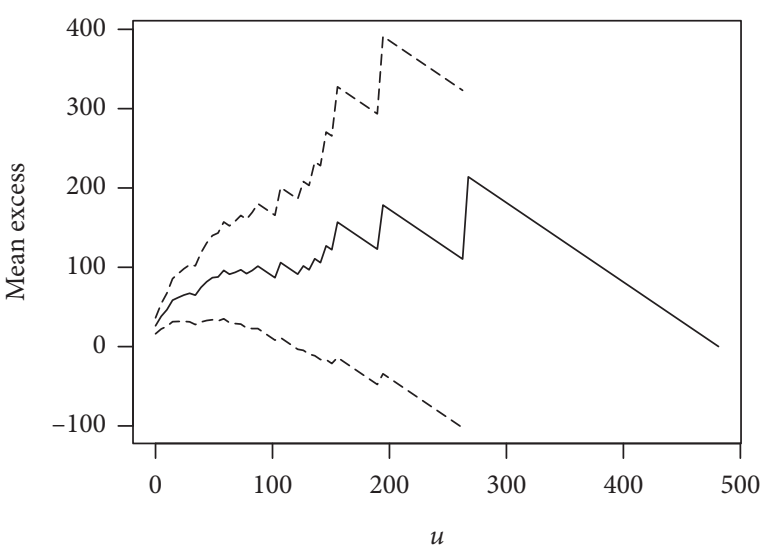

Figure 2: Mean excess plot of damage areas.

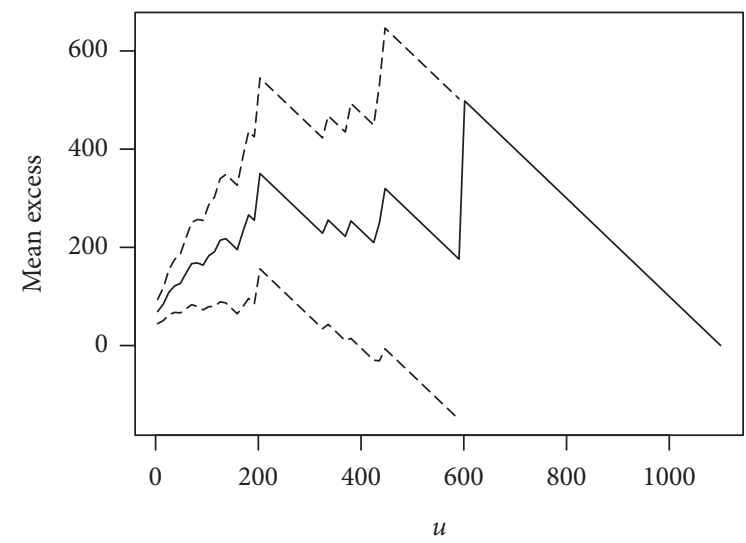

Figure 3: Mean excess plot of deaths.

that the plot curves up until around the value of 48 and straightens after that. Based on the mean excess plot, one might select the value of 48 as a threshold.

In order to check the validity of the threshold, we select a series of threshold values and employ the maximum likelihood estimation to get a series of parameters. Clearly, Figure 4 indicates that shape parameter $\xi$ and scale parameter $\sigma$ are stable around the threshold of 48. By using similar arguments to the above and referring to Figures 3 and 5 , an appropriate threshold value of deaths is 88 .
After calculating the threshold values, the maximum likelihood method will be adopted to estimate the rest of the parameters. Estimates are given in Table 1.

The diagnostic plots for assessing the accuracy of the POT model fitted to the damage areas and deaths are shown in Figures 6 and 7 , respectively. Observing the probability plot and Q-Q plot in Figure 6, each set of plotted points is near-linear. There it should be noted that a largest catastrophe, the 2009 summer severe flood in Sichuan Province, is extreme in the China history. Due to this fact, one concludes that the diagnostic plots give a good fit for the damage areas. Similar analysis can be conducted for deaths from Figure 7.

3.3. Selection of Copula Function and Parameters Estimation. Copula function is called joint function, which plays the role in the relationship between multivariate distribution function and its marginal distributions. Copula function can be classified into two categories, namely, elliptic Copula family and Archimedean Copula family. The latter is an important Copula family that has a wide range of applications. There are a number of reasons for its popularity, such as the great variety of families and the many nice properties; see $[26,27]$ for a detailed description. Therefore, we shall adopt three common Archimedean Copula functions (Clayton Copula, Gumbel Copula, and Frank Copula) to construct related studies.

First of all, we plug the parameters of the POT model in Table 1 into equation (7). Then, the distribution series $\left\{u_{i}, v_{i}\right\}$ whose values belong to $[0,1]$ can be obtained via probability integral transform. By using the box-test, we can discover that the series after transforming are independent. Furthermore, let the series $\left\{u_{i}, v_{i}\right\}$ be the observations of Copula and carry out parameters' estimation by the maximum likelihood method. Since different kinds of Copula functions possess different dependent patterns, it is very significant to pick out the suitable Copula function to depict dependence structure. To proceed, we employ both Kolmogorov-Smirnov (K-S) test and Q-Q plot to test the appropriateness of Copula model selection. It is worth pointing out here that the K-S test is available without any assumption on the distribution of data. The results from Figure 8 indicate that Clayton Copula provides a better fit for the data compared to 


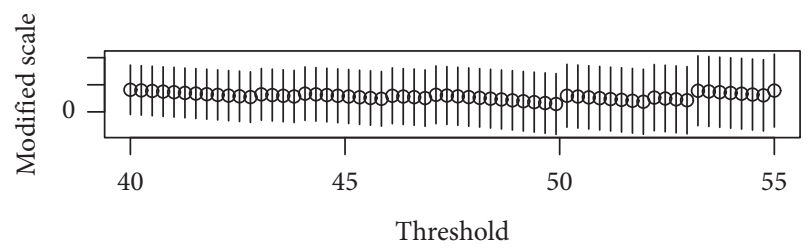

(a)

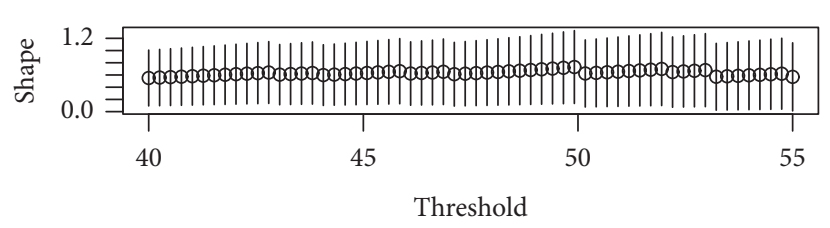

(b)

FigURE 4: Figure of calibration scale and shape parameters of damage areas.

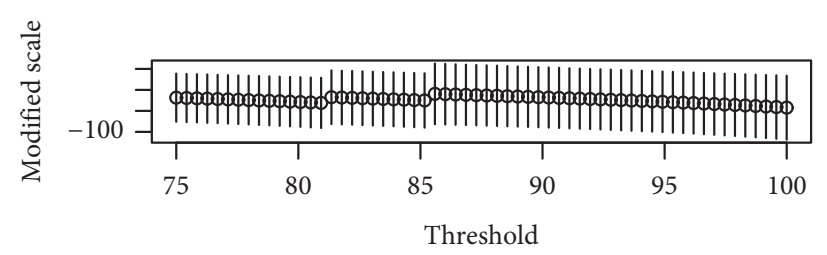

(a)

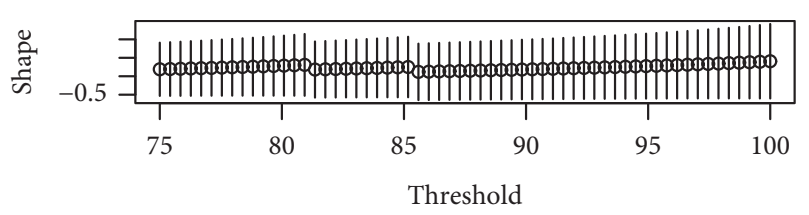

(b)

FIGURE 5: Figure of calibration scale and shape parameters of deaths.

TABle 1: POT parameters' estimates.

\begin{tabular}{lcccc}
\hline Parameters & $\xi$ & $\sigma$ & $u$ & $N_{u}$ \\
\hline Damage areas & 0.2040 & 70.0527 & 48 & 16 \\
Deaths & 0.4953 & 95.0446 & 88 & 25 \\
\hline
\end{tabular}

the other Copula functions. Moreover, Table 2 shows that the K-S test statistics of Clayton Copula at the 0.01 significance level is 0.0443 and its $p$ value is 0.9563 , from which we further know that only Clayton Copula passes the K-S test.

\section{Numerical Example and Simulation}

4.1. Simulation and Valuing for the Catastrophe Reinsurance Model. In this subsection, we will concentrate on determining the value of flood reinsurance. Before estimation, some related parameter values need to be set. According to the relative data of the real insurance market, the basic parameter values are given in Table 3.

Recalling that the explicit solution of (1) is difficult to compute, we estimate the value of reinsurance by Monte Carlo simulation technique. The steps of simulation can be explicitly outlined as follows:

(1) Simulate the interarrival times $\left\{\tau_{j}, j \geq 1\right\}$ from an exponential distribution with mean value $1 / \lambda$, provided that the occurrence of events is a Poisson process with intensity $\lambda$. Denote the number of events by $M=M(T)=M((0, T])$. If $M=0$, let total loss be 0 and continue to the next simulation; otherwise, denote the times of events by $t_{j}$; namely, $t_{j}=$ $\sum_{i=1}^{j} \tau_{i}(j=1,2, \ldots, M)$.

(2) Generate $M$ pairs of random numbers $\left(w_{i}, v_{i}\right)$ from Clayton Copula function.

(3) From marginal distribution, calculate the observations about losses and deaths. They are denoted by $\left\{x_{i}, y_{i}\right\}$; that is, $x_{i}=F_{X}^{-1}\left(w_{i}\right)$ and $y_{i}=F_{Y}^{-1}\left(v_{i}\right)$.
(4) Simulate the interest rate path within $(0, T]$. In this end, divide $(0, T]$ into $10000 T$ equal parts and replace (2) by the following discretization scheme:

$$
\begin{aligned}
r(i)-r(i-1)= & \alpha(\mu-r(i-1)) \mathrm{d} t \\
& +\beta \sqrt{r(i-1)} \varepsilon_{i}, \quad i=1,2, \ldots, 10000 T,
\end{aligned}
$$

where $r(0)=r_{0}$ and $\varepsilon_{i} \sim N(0, \Delta t)$ with $\triangle t=10^{-4}$. Next, generate the numbers $\varepsilon_{1}, \varepsilon_{2}, \ldots, \varepsilon_{10000 T}$ from an $N(0, \Delta t)$ distribution, and then plug them into equality (8). Based on this iteration method, we may obtain one simulation of interest rate path and further derive the discount factor which now can be approximately expressed by $e^{-\int_{0}^{t_{j}} r(t) \mathrm{d} t} \approx e^{-\sum_{i=1}^{M} r}$ $\left(t_{i}\right) \triangle t$.

(5) Substitute the results of procedures (1)-(4) into (1), and then a simulation result of the value of catastrophe reinsurance is obtained.

(6) Repeat the procedures (1)-(5) for $K$ times, and calculate the mean value.

To determine the value, we run $10^{5}$ simulations and find that the catastrophe reinsurance value $P=87.605$ million dollars.

\subsection{Sensitivity Analysis for the Catastrophe Reinsurance}

4.2.1. The Effects of Initial Interest Rate Value $r_{0}$. Value of catastrophe reinsurance decrease if the initial value of interest rate $r_{0}$ increases, as indicated in Table 4 . This obviously satisfies the rule of the real interest rate markets. The higher the initial value of interest rate is, the less the interest value after interest rate discount is. On the other hand, average interest rate in the long run $\mu$ also shows the inverse relation with catastrophe reinsurance value. This effect is especially 

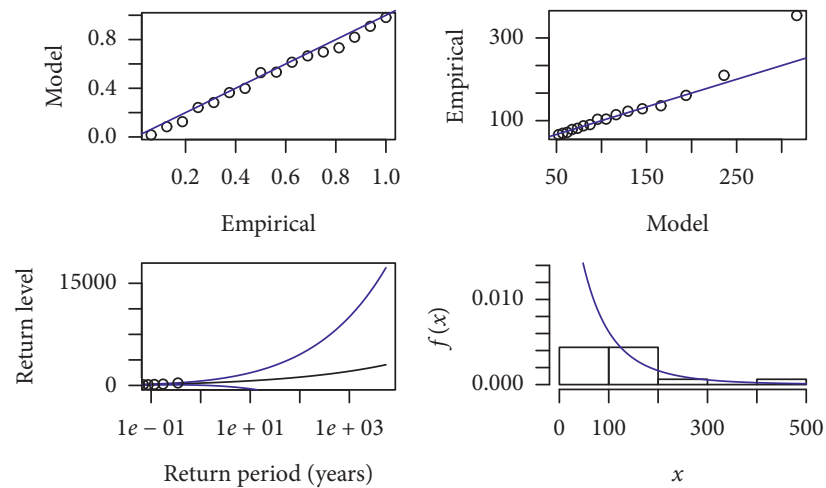

Figure 6: Diagnostic plots of damage areas. (a) Probability plot. (b) Quantile plot. (c) Return level plot. (d) Density plot.
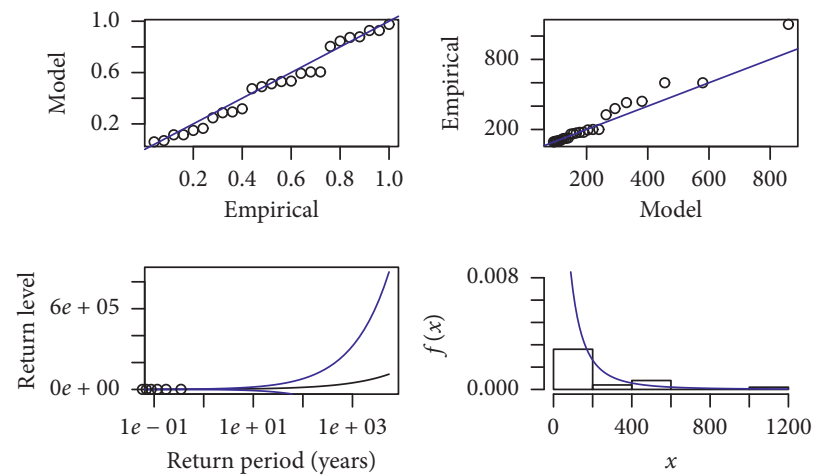

Figure 7: Diagnostic plots of deaths. (a) Probability plot. (b) Quantile plot. (c) Return level plot. (d) Density plot.

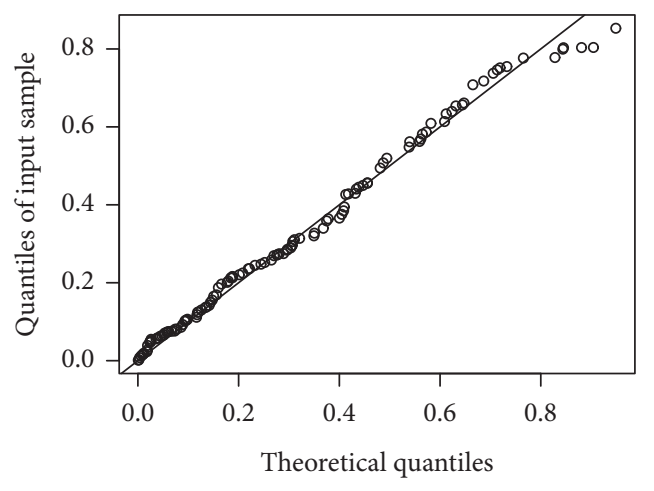

(a)

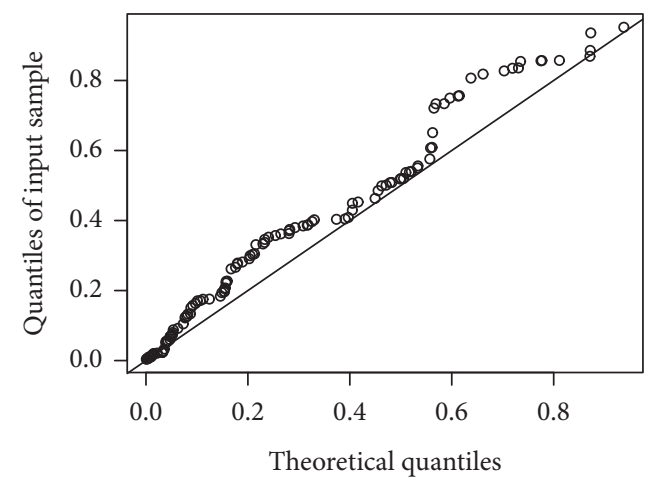

(b)

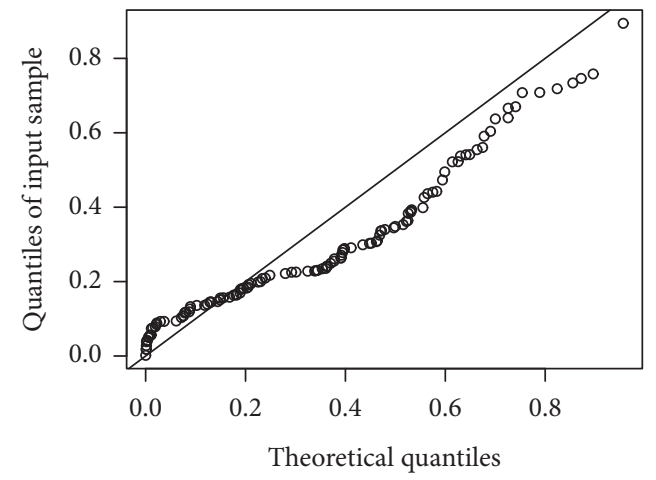

(c)

Figure 8: Q-Q plots of Clayton Copula (a), Gumbel Copula (b), and Frank Copula (c). 
TABle 2: Parameter estimates of Copula function and K-S test results.

\begin{tabular}{lccc}
\hline Copula function & Clayton Copula & Gumbel Copula & Frank Copula \\
\hline$\theta$ value & 0.5485 & 1.3440 & 2.6690 \\
K-S value & 0.0443 & 0.1627 & 0.6221 \\
$p$ value & 0.9563 & 0.0022 & $\leq 0.01$ \\
\hline
\end{tabular}

TABLE 3: Basic parameter values.

\begin{tabular}{lcc}
\hline Text interpretation & Symbol & Value \\
\hline Initial interest rate value & $r_{0}$ & 0.03 \\
Speed of mean-reverting & $\alpha$ & 0.04 \\
Long run interest rate mean & $\mu$ & 0.03 \\
Volatility of the interest rate & $\beta$ & 0.02 \\
Attachment point & $D$ & $T$ \\
Maturity time (year) & $T$ & 1 \\
Claim coefficient of damage areas (sq. km) & $c_{1}$ & 168.49 million dollars \\
Claim coefficient of deaths (one person) & $c_{2}$ & 1 million dollars \\
\hline
\end{tabular}

TABLE 4: Effect of initial interest rate value.

\begin{tabular}{lccc}
\hline$r_{0}$ & 0.03 & 0.06 & 0.09 \\
\hline$P$ & 87.605 & 86.304 & 83.057 \\
\hline
\end{tabular}

TABle 5: Effect of dependence $\theta$.

\begin{tabular}{lccccccc}
\hline Quantile & Mean & 0.5 & 0.75 & 0.9 & 0.95 & 0.99 & 0.9999 \\
\hline$\theta=0.5485$ & 87.605 & 11.656 & 10.368 & 250.793 & 378.791 & 779.679 & 7100.907 \\
$\theta=2.5485$ & 93.532 & 13.952 & 112.451 & 264.075 & 403.031 & 805.368 & 6051.593 \\
$\theta=4.5485$ & 96.980 & 14.739 & 117.958 & 275.371 & 414.009 & 844.241 & 7098.610 \\
\hline
\end{tabular}

Note: the simulation times are 100,000 .

pronounced in the case of a longer period of time. In view of that, the computation results of the value of catastrophe reinsurance with different $\mu$ are similar to those in the case of $r_{0}$, and the computation results between $\mu$ and reinsurance value are omitted for simplicity.

4.2.2. The Effect of Dependence $\theta$. The relationship between $\theta$ and the value of catastrophe reinsurance is explicitly shown in Table 5. The value increases with $\theta$. A possible explanation of the results may be related to the fact that a larger $\theta$ implies more dependence; that is, the dependence between catastrophe damage areas and deaths increases. In other words, the probability of claim exceeding the attachment point becomes bigger. Consequently, the value of reinsurance is expected to rise. However, due to the extreme heavy tail of the GPD, the results in the extreme right tail are not exactly the same each time. In all, we can confirm that the dependence among catastrophe reinsurance claims has significant effect on the value.

\section{Conclusions}

This study is the first to value multirisk claims catastrophe reinsurance contract under the stochastic interest rate. In view of the heavy-tail features and dependence of the catastrophe variables, POT model and Copula model are combined to describe them. Moreover, we also apply the CIR model to depict the characteristic of interest rates market. Consequently, using Monte Carlo simulations, we give the value of multirisk catastrophe reinsurance contract and perform a sensitivity analysis. This new framework established in this paper for valuing multirisk claims catastrophe reinsurance contract may be interesting to (re)insurance companies and other financial institutions that want to transfer catastrophic risks. We also remark that although this paper mainly focuses on the flood hazard, this framework also works for other catastrophe hazards. The framework put forward in this paper not only enriches existing research on catastrophe reinsurance values but also provides theoretical guidance for insurers to value the 
multirisk catastrophe reinsurance, which has an important reference value.

The sensitivity analysis performed in this paper indicates that the catastrophe reinsurance value has an inverse relation with initial value of interest rate and average interest rate in the long run. In addition, a high level of dependence between catastrophe variables increases the catastrophe reinsurance value, from which we conclude that interest rate structure and the dependence between different kinds of catastrophe variables play important roles in valuing the catastrophe reinsurance contract.

\section{Data Availability}

The data can be obtained from http://www.dartmouth.edu/ floods/Archives/index.html.

\section{Conflicts of Interest}

The author declares that there are no conflicts of interest.

\section{Acknowledgments}

This work was supported by General Planning Project of Fujian Social Science Association (no. fj2018b032).

\section{References}

[1] P. Strickler, "Rückversicherung des kumulrisikos in der lebensversicherung," in Proceedings of the XVI International Congress of Actuaries, pp. 666-679, Brussels, Belgium, February1960.

[2] M. Harbitz, "Catastrophe covers in life assurance," Transactions of International Congress of Actuaries, vol. 14, no. 1, pp. 109-113, 1992.

[3] E. Ekheden and O. Hössjer, "Pricing catastrophe risk in life (re)insurance," Scandinavian Actuarial Journal, vol. 2014, no. 4, pp. 1-16, 2014.

[4] M. Leppisaari, "Modeling catastrophic deaths using EVT with a microsimulation approach to reinsurance pricing," Scandinavian Actuarial Journal, vol. 2016, no. 2, pp. 113-145, 2016.

[5] K. Antonio and R. Plat, "Micro-level stochastic loss reserving for general insurance," Scandinavian Actuarial Journal, vol. 2014, no. 7, 145 pages, Article ID 755938, 2014.

[6] M. Pigeon, K. Antonio, and M. Denuit, "Individual loss reserving with the multivariate skew normal framework," Astin Bulletin, vol. 43, no. 3, pp. 399-428, 2013.

[7] C. D. Daykin, T. Pentikäinen, and M. Pesonen, Practical Risk Theory for Actuaries, Chapman and Hall, London, UK, 1994.

[8] M. Egami and V. R. Young, "Indifference prices of structured catastrophe (CAT) bonds," Insurance: Mathematics and Economics, vol. 42, no. 2, pp. 771-778, 2008.

[9] M. N. Giuricich and K. Burnecki, "Modelling of left-truncated heavy-tailed data with application to catastrophe bond pricing," Physica A: Statistical Mechanics and Its Applications, vol. 525, pp. 498-513, 2019.

[10] M. Romaniuk, "Analysis of the insurance portfolio with an embedded catastrophe bond in a case of uncertain parameter of the insurer's share. information systems architecture and technology," Advances in Intelligent Systems and Computing, vol. 524, pp. 33-43, 2017.
[11] H. Yang and L. Zhang, "Optimal investment for insurer with jump-diffusion risk process," Insurance: Mathematics and Economics, vol. 37, no. 3, pp. 615-634, 2005.

[12] P. Nowak and M. Romaniuk, "Pricing and simulations of catastrophe bonds," Insurance: Mathematics and Economics, vol. 52, no. 1, pp. 18-28, 2013.

[13] Z. Ma, C. Ma, and S. Xiao, "Pricing zero-coupon catastrophe bonds using EVT with doubly stochastic poisson arrivals," Discrete Dynamics in Nature and Society, vol. 2017, Article ID 3279647, 14 pages, 2017.

[14] X. Chen and W. Zhuo, "Martingale and duality methods for optimal investment and reinsurance problem in a Lévy model," Communications in Statistics-Theory and Methods, vol. 49, no. 23, pp. 5738-5764, 2020.

[15] W.-S. Chan, H. Yang, and L. Zhang, "Some results on ruin probabilities in a two-dimensional risk model," Insurance: Mathematics and Economics, vol. 32, no. 3, pp. 345-358, 2003.

[16] X. Shen and Y. Zhang, "Ruin probabilities of a two-dimensional risk model with dependent risks of heavy tail," Statistics \& Probability Letters, vol. 83, no. 7, pp. 1787-1799, 2013.

[17] S. Wang and W. Wang, "Precise large deviations for sums of random variables with consistent variation in dependent multi-risk models," Communications in Statistics - Theory and Methods, vol. 42, no. 24, pp. 4444-4459, 2013.

[18] W. Huang, C. Weng, and Y. Zhang, "Multivariate risk models under heavy-tailed risks," Applied Stochastic Models in Business and Industry, vol. 30, no. 3, pp. 341-360, 2014.

[19] J. Li and H. Yang, "Asymptotic ruin probabilities for a bidimensional renewal risk model with constant interest rate and dependent claims," Journal of Mathematical Analysis and Applications, vol. 426, no. 1, pp. 247-266, 2015.

[20] D. G. Konstantinides and J. Li, "Asymptotic ruin probabilities for a multidimensional renewal risk model with multivariate regularly varying claims," Insurance: Mathematics and Economics, vol. 69, pp. 38-44, 2016.

[21] J. C. Cox, J. E. Ingersoll, and S. A. Ross, "A theory of the term structure of interest rates," Econometrica, vol. 53, no. 2, pp. 385-407, 1985.

[22] J. Pickands, "Statistical inference using extreme order statistics," The Annals of Statistics, vol. 3, no. 1, pp. 119-131, 1975.

[23] A. A. Balkema and L. De Haan, "Residual life time at great age," The Annals of Probability, vol. 2, no. 5, pp. 792-804, 1974.

[24] J. Pickands, "The two-dimensional possion process and external process," The Annals of Statistics, vol. 8, no. 1, pp. 745-756, 1971.

[25] P. Embrechts, A. Höing, and A. Juri, "Using copulae to bound the Value-at-Risk for functions of dependent risks," Finance and Stochastics, vol. 7, no. 2, pp. 145-167, 2003.

[26] H. Joe, Multivariate Models and Dependence Concepts, Chapman and Hall, London, UK, 1997.

[27] R. Nelsen, An Introduction to Copulas, Springer Verlag, Berlin, Germany, 1999. 